\title{
A DEVICE FOR GENERATING FIELDS OF EVEN CLASS NUMBER
}

\section{HARVEY COHN ${ }^{1}$}

The device in question is typefied by the following two theorems:

QUADRATIC THEOREM. If for integral $g(>0), m, k(>1)$

$$
m=\frac{k^{2}-1}{g}+g,
$$

and $f=m^{2}+4$ is square-free, then the quadratic field of discriminant $f$ has even class number.

CUBIC THEOREM. If for integral $g(>0), m, k(>1)$,

$$
m=\frac{k^{2}-2 g-1}{g(g+1)}+g-1,
$$

and $f=m^{2}+3 m+9$ is a square-free $e^{2}$ integer, then an abelian cubic field of discriminant $f^{2}$ has even class number.

PROOF OF THEOREMS.

$$
\begin{array}{ccc}
\text { Quadratic } & * & \text { Cubic } \\
n=2 & * & n=3 .
\end{array}
$$

We consider a square-free number $f$ of the form

$$
f=m^{2}+4, \quad * \quad f=m^{2}+3 m+9,
$$

with $m>0$, together with the equation $F(\xi)=0$, where

$F(x)=x^{2}-m x-1 . \quad * \quad F(x)=x^{3}-m x^{2}-(m+3) x-1$.

The equation has (root) discriminant

$$
\prod_{i>j}\left(\xi_{i}-\xi_{j}\right)^{2}=f^{n-1}
$$

where $\xi_{1}, \cdots, \xi_{n}$ are the roots which generate the abelian field $R(\xi)$.

Received by the editors August 23, 1955.

1 Presented June 24, 1955 as an invited address to the National Science Foundation Research Conference on The Theory of Numbers at Pasadena under the title Accessibility of algebraic numbers with rounded norms. The author is indebted to Drs. S. Rosen and A. W. Jacobson of the Wayne University Computation Center for making the UDEC available for the computation of the table.

2 If 9 divides $f$ the hypothesis can be broadened to include values of $f$ for which $f / 3$ is square-free, by a slight modification of the proof. Compare footnote 3 . 
The quantity $f^{n-1}$ is also the discriminant of the field $R(\xi)$, as follows from the fact that $f$ is square-free while

$$
R(\xi) \quad * \quad R(\xi, \rho),\left[\rho^{2}+\rho+1=0\right]
$$

is formed by the adjunction of the irreducible radical ${ }^{3}$

$$
f^{1 / 2} \text { to } R \quad * \quad[f(m-3 \rho)]^{1 / 3} \text { to } R(\rho) \text {. }
$$

As a result, the most general integer in $R(\xi)$ is given by the expres$\operatorname{sion} \lambda=A_{0}+\cdots+A_{n-1} \xi^{n-1}$ where $A_{0}, \cdots, A_{n-1}$ are rational integers. It also follows, by subtraction, that no two of the $n$ conjugates $\xi_{1}-g, \cdots, \xi_{n}-g$ can have as common factors different conjugates of a prime ideal. Thus any number of the form $\xi-g$ with square norm must generate a square ideal. We finally note, in preparation, that a unit which is totally positive (i.e., for which all three conjugates are positive) is the square of another unit in $R(\xi)$. To see this, we need only display the following $2^{n}$ units which exhibit all possible $2^{n}$ arrays of + and - signs when their $n$ conjugates are listed:

$+1,-1,+\xi_{1},-\xi_{1} . \quad * \quad+1,-1,+\xi_{1},-\xi_{1},+\xi_{2},-\xi_{2},+\xi_{3},-\xi_{3}$.

In fact, in some order of listing,

$$
\begin{array}{rlrl}
m<\xi_{1}<m+1, & * & m+1<\xi_{1}<m+2, \\
-1<\xi_{2}<0 . & * & -1<\xi_{2}<0, \\
& * & -2<\xi_{3}<-1 .
\end{array}
$$

We consider now integers $g, k$ such that

$$
F(g)=N(g-\xi)=-k^{2},
$$

This result leads on expansion to equations (1), (2). Then,

$$
0<g<m+1 ; \quad * \quad 0<g<m+2 ;
$$

and the number, of norm $k^{2}$,

$$
\zeta=\xi(\xi-g)
$$

must be totally positive. Thus $\xi-g$ generates the square of an ideal which could not be principal unless $\zeta$ were a perfect square. We shall now eliminate this last possibility, completing the proofs.

In the quadratic case, the equation $\left(A_{0}+A_{1} \xi\right)^{2}=\xi(\xi-g)$ leads to a triviality, all the more so in the cubic case $\left(A_{2}=0\right)$. Henceforth we

${ }^{3}$ This argument, superfluous when $f$ is prime, uses the only nonelementary result here. See H. Hasse, Arithmetiche Bestimmung von Grundeinheit und Klassenzahl in zyklischen, kubischen und biquadratischen Zahlkörpern, Berlin, 1950. 
restrict ourselves to the cubic case, writing in terms of three conjugates

$$
A_{0}+A_{1} \xi_{i}+A_{2} \xi_{i}^{2}= \pm \zeta_{i}^{1 / 2} \quad(i=1,2,3),
$$

with choice of sign open. We solve for $A_{2}$, recalling that $f^{2}$ is the (root) discriminant, so that

$$
f A_{2}= \pm \zeta_{1}^{1 / 2}\left(\xi_{2}-\xi_{3}\right) \pm \zeta_{2}^{1 / 2}\left(\xi_{3}-\xi_{1}\right) \pm \zeta_{3}^{1 / 2}\left(\xi_{1}-\xi_{2}\right)
$$

We find, from equations (4) and (5) that

$$
\begin{array}{ll}
\left|\zeta_{1}\right|<(m+1)^{2}, & \left|\xi_{2}-\xi_{3}\right|<2, \\
\left|\zeta_{2}\right|<(m+1), & \left|\xi_{3}-\xi_{1}\right|<m+4, \\
\left|\zeta_{3}\right|<2 m, & \left|\xi_{1}-\xi_{2}\right|<m+3,
\end{array}
$$

so that from equation (6),

$$
\left|A_{2}\right| \leqq\left[2(m+1)+\left(1+2^{1 / 2}\right)(m+1)^{1 / 2}(m+4)\right] /\left(m^{2}+3 m+9\right) .
$$

Thus if $m \geqq 12, A_{2}=0$, leading to a triviality. (The smaller $m$ can be checked by hand as the values of $g$ are limited by equation (4) and the values of $k$ by equation (2).)

COROLlaRY. If $g \equiv 0 \bmod 4$, then the fields $R(\xi)$ referred to in the above theorems have an unramified quadratic extension produced by $[\xi(\xi-g)]^{1 / 2}$.

The proofs depend on the relation ${ }^{4} \zeta \equiv \xi^{2} \bmod 4$. We should note that with $g \equiv 0 \bmod 4$ the condition " $g(>0)$ " in the (cubic) theorems is superfluous from the integral nature of $m$, as $k^{2} \not=-1 \bmod (g+1)$.

Tabulation. The above proofs still leave unanswered the question of how effective a method we have for (say) finding cubic fields of even class number where the discriminant is $f^{2}$, for $f\left(=m^{2}+3 m+9\right)$ restricted to prime values. Under such a restriction we have a convenient basis of comparison in Mr. Peter Swinnerton-Dyer's unpublished table (abbreviated SDT) of class numbers as computed from the cyclotomic units (rather than the ideal structure). The table below lists $g, k, m, f$ and the class number $h$ (where provided by SDT) for $f<100,000, m<316, g \equiv 0 \bmod 4$. The first field of even class number that does not emerge has $f=18,913$ with $h=100$ according to SDT.

4 See D. Hilbert, Über die Theorie der relativ-Abelschen Zahlkörper, Acta Math. vol. 26 (1902) pp. 99-132. 
We now have another illustration of the classical observation that the composite norms (such as $-k^{2}$ ) which are so useful in determining class structures ${ }^{5}$ are more readily accessible than one would believe on the basis of known theory alone. In this case the desired norms are found from the generating polynomial, i.e., $F(g)$, without requiring the use of the full (ternary) norm expression for the general integer of the field. This convenient state of affairs, as expected, becomes dissipated as $f \rightarrow \infty$, however.

TABLE OF UNRAMIFIED QUADRATIC EXTENSIONS OF CUBIC FIELDS OF EVEN CLASS NUMBER AND PRIME CONDUCTOR $f=m^{2}+3 m+9<100,000$, FORMED BY ADJOINING $[\xi(\xi-g)]^{1 / 2}$, OF NORM $k$, WHERE $\xi^{3}-m \xi^{2}-(m+3) \xi-1=0$.

\begin{tabular}{|rrrrr|}
\hline$g$ & $k$ & $m$ & \multicolumn{1}{c|}{$f$} & \multicolumn{1}{c|}{$h$} \\
\hline 4 & 13 & 11 & 163 & 4 \\
12 & 5 & 11 & 163 & 4 \\
4 & 17 & 17 & 349 & 4 \\
12 & 31 & 17 & 349 & 4 \\
24 & 7 & 23 & 607 & 4 \\
16 & 47 & 23 & 607 & 4 \\
12 & 47 & 25 & 709 & 4 \\
4 & 23 & 29 & 937 & 4 \\
28 & 41 & 29 & 937 & 4 \\
24 & 157 & 64 & 4,297 & 16 \\
24 & 193 & 85 & 7,489 & $?$ \\
4 & 43 & 95 & 9,319 & 28 \\
84 & 293 & 95 & 9,319 & 28 \\
40 & $11 \cdot 29$ & 101 & 10,513 & 64 \\
60 & 499 & 127 & 16,519 & 52 \\
72 & 557 & 130 & 17,299 & 52 \\
24 & 257 & 133 & 18,097 & 52 \\
\hline
\end{tabular}

\begin{tabular}{|rcccc|}
\hline \multicolumn{1}{|c}{$g$} & $k$ & $m$ & $f$ & $h$ \\
\hline$\ldots$ & $\ldots$ & 136 & 18,913 & 100 \\
72 & $13 \cdot 47$ & 142 & 20,599 & 112 \\
4 & 53 & 143 & 20,887 & 64 \\
28 & 307 & 143 & 20,887 & 64 \\
64 & 577 & 143 & 20,887 & 64 \\
124 & 557 & 143 & 20,887 & 64 \\
144 & 17 & 143 & 20,887 & 64 \\
36 & $7 \cdot 59$ & 163 & 27,067 & $?$ \\
156 & 443 & 163 & 27,067 & $?$ \\
144 & $11 \cdot 67$ & 169 & 29,077 & $?$ \\
168 & 239 & 169 & 29,077 & $?$ \\
88 & $5 \cdot 167$ & 176 & 31,513 & $?$ \\
100 & $29 \cdot 31$ & 179 & 32,587 & $?$ \\
180 & 19 & 179 & 32,587 & $?$ \\
88 & 1,013 & 218 & 48,187 & $?$ \\
84 & $11 \cdot 107$ & 277 & 77,569 & $?$ \\
112 & 1,567 & 305 & 93,949 & $?$ \\
\hline
\end{tabular}

STANFORD UNIVERSITY

- Compare H. Cohn, Some experiments in ideal factorization on the MIDAC, J. Assoc. Comput. Mach. vol. 2 (1955) pp. 111-116. 